\title{
Heat Transfer Model of Multilayer Thermal Protective Clothing for High-Temperature Operation
}

\author{
Man-Jing $\mathrm{Li}^{1}$, Mao Zhu ${ }^{1}$, Jia-Xu $\operatorname{Han}^{1} \&$ Yuan-Biao Zhang ${ }^{1,2,3}$ \\ ${ }^{1}$ Mathematical Modeling Innovative Practice Base, Jinan University, Zhuhai Campus, Zhuhai 519070, China \\ ${ }^{2}$ Packaging Engineering Institute, Jinan University, Zhuhai Campus, Zhuhai 519070, China \\ ${ }^{3}$ Key Laboratory of Product Packaging and Logistics of Guangdong Higher Education Institutes, Jinan \\ University, Zhuhai Campus, Zhuhai 519070, China \\ Correspondence: Yuan-Biao Zhang, Packaging Engineering Institute, Jinan University, Zhuhai Campus, Zhuhai \\ 519070, China. E-mail: zybt@jnu.edu.cn
}

Received: September 2, 2019

Accepted: October 4, 2019

Online Published: October 5, 2019

doi:10.5539/mas.v13n11p21

URL: https://doi.org/10.5539/mas.v13n11p21

\begin{abstract}
The thermal protective clothing for high-temperature operation usually consists of three-layer fabrics and a gap called the air layer or Layer IV between Layer III and skin. In order to design more effective thermal protective clothing at less cost, based on the heat transfer principles, we establish heat transfer models of fabrics and air layer, which are one-dimensional nonlinear partial differential equations with constant coefficients. In the three-layer fabrics, we consider the effects of heat conduction and heat radiation in Layer I but only consider heat conduction in Layer II and Layer III. Furthermore, the heat transfer model of Layer IV is decoupled and simplified to steady-state heat conduction in Layer IV and radiation heat transfer on surface of Layer IV. According to the explicit difference schemes for the models, we use the parameters in an experiment which puts a thermal manikin in high-temperature environment for some time and measures the temperature of lateral skin at regular time, to solve the models and calculate the temperature of each layer. With MATLAB, the visual interface of three-dimensional temperature distribution is provided, which is reference for functional design of thermal protective clothing. We also compare the simulation result of skin surface with the experimental data. The results show that at the same position, the temperature rises over time but with decreasing rate and finally reaches the steady state. Moreover, at one moment after reaching the steady state, the temperature has a gradual decrease with the increase of distance from the external environment.
\end{abstract}

Keywords: multilayer thermal protective clothing, heat transfer, finite difference method

\section{Introduction}

The thermal protective clothing for high-temperature operation, which is normally used in high-temperature or ultra-high-temperature environment, can reduce the amount of heat accumulated near human skin to avoid the harm of heat sources to human body such as burns by slowing down the rate of heat transfer. It not only has the properties of ordinary protective clothing, but also possesses some characteristics such as fire resistance, liquid repellency, no droplet during combustion and safety and comfort when heated, so it is often used in petroleum industry, chemical industry, metallurgy, fire protection, national defense and the places having open fire, electric spark, molten metal and flammable substance. But according to some studies, even when people wear the thermal protective clothing, extremely high-temperature environment may cause skin burns or burns. To improve this problem, many researchers have done a lot of research to evaluate the performance of thermal protective clothing and the majority of the physical protective clothing experiment is based on high-temperature environment. However, the experimental material can not be reused after the experiment, which results in high cost and unnecessary waste of resources. Therefore, it is necessary to establish a heat transfer model of thermal protective clothing for high-temperature operation, which can offer theoretical reference on the design of the clothing and provide more scientific security for human body through simulation experiment.

Because the establishment of this heat transfer model has great benefits for the study of its performance, this topic has gradually become a new research focus in recent years. According to the number of material layers, the mathematical models of thermal protective clothing are mainly divided into monolayer model and multilayer 
model. The monolayer model only involves a shell of clothing and researchers mainly study the effects of heat radiation of external environment, physical properties of fabric, moisture inside fabric and the thickness of air layer on the protective performance. Gibson (1996) proposed a multiphase heat and mass transfer model, but the radiant heat in fabric layer is not taken into account. Then, Torvi (1997) introduced the heat transfer model of outer fabric under the environment of strong radiation or long-term low radiation. Based on the models established by Gibson and Torvi, a coupled heat and moisture model with porous media for thermal protective clothing was developed (Chitrphiromsri \& Kuznetsov, 2005). In this model, they considered the sweat on the surface of the skin, which can evaporate or be absorbed by fibers, and they used the parameter estimation method to calculate the gas temperature around human body and convection heat conductivity. Song Guowen et al. (2008) analyzed the heat and moisture transfer model of thermal protective clothing under flash fire conditions and got the distribution of temperature and water vapor in fabric and air layer by numerical simulations. In addition, they used the model of skin to evaluate thermal damage and the results showed that the thickness of the air layer had a crucial impact on the performance of thermal protective clothing. On the base of the previous study, Ghazy et al. $(2010 ; 2013 ; 2014)$ used the empirical formulas to transform the physical parameters of fabric from constant to variable, which made the simulation results more precise. Besides, they proposed a simple and accurate model of air layer and a heat transfer model of monolayer fabric in motion. According to the monolayer model, many researchers also study the multilayer moisture and heat transfer model of thermal protective clothing, Mell and Lawson (2000) firstly established a heat transfer model between layers of multilayer fabric, which is develop from Torvi's model. Because phase change materials have an influence on thermal protection performance of clothing, a multilayer dynamic heat transfer model with phase change materials was built (Mercer \& Sidhu, 2008; Elgafy \& Mishra, 2014). Considering the effect of moisture on thermal protection, Lawson et al. (2010) made continued developments in protective clothing modeling of multilayer composite state under instantaneous conditions. Moreover, taking the impact of air layer into account, Ghazy and Bergstrom (2012) further improved the multilayer heat and moisture transfer model by introducing multi air layers.

According to the classic heat transfer model and existing research, we firstly propose heat transfer models of multilayer thermal protective clothing for high-temperature operation. In these models, we consider the heat conduction and heat radiation in Layer I while there is only heat conduction in Layer II and Layer III. In addition, to simplify calculation, we decouple the model of Layer IV. After determining the initial and boundary condition, using the finite difference method and the models we establish, we carry out numerical simulation with the parameters of one thermal manikin experiment. Finally, the temperatures of each layer, which are varying with time and thickness, can be calculated and the temperature distribution can be plotted by MATLAB. We assume the parameters and physical structure of three-layer fabrics remain unchanged and take no account of the moisture transfer and heat exchange in skin. Besides, the direction of heat transfer is assumed to be perpendicular to the skin, which means that our models are one-dimensional. Our models' purpose is to get insight law of heat transfer within textiles and offer reasonable and scientific basis for the design of thermal protective clothing, which can help the researchers develop more effective thermal protective clothing at less cost.

\section{Method}

\subsection{Establishment of Heat Transfer Model}

When working in high-temperature environment, people need to wear thermal protective clothing to avoid burns. Thermal protective clothing usually consists of three-layer fabrics, which is regarded as Layer I, Layer II and Layer III. Layer I is in contact with the external environment. There is a space between Layer III and skin, which is regarded as Layer IV or air layer. A sectional view of the environment, three-layer fabrics, air layer and skin is shown in Figure 1. 


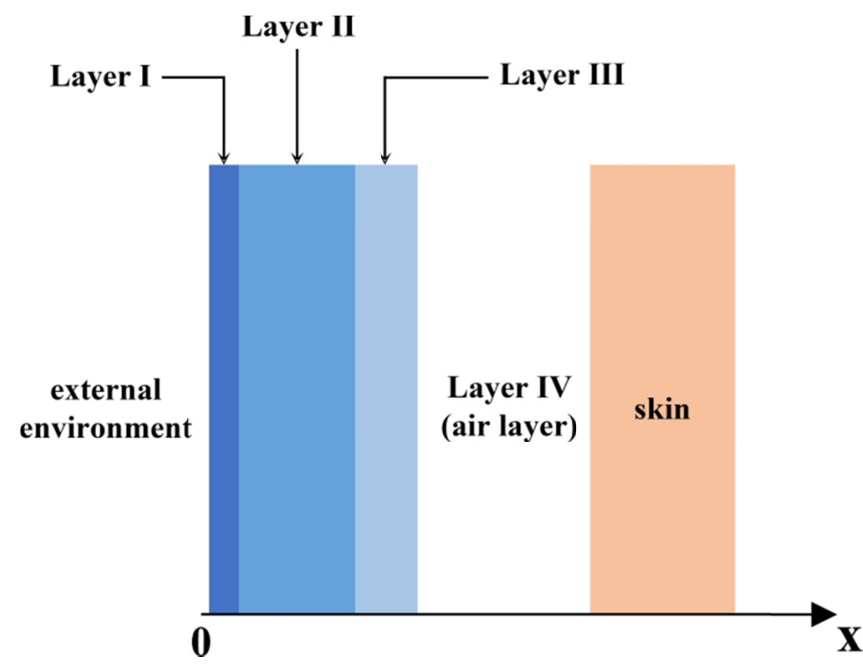

Figure 1. Sectional view of the environment, three-layer fabrics, air layer and skin

According to Pan Bin (2016), there are two main forms of heat transfer in the heat transfer model, heat conduction and heat radiation. When there is a temperature difference between two media, heat transfer occurs, which we call it heat conduction. Besides, heat radiation refers to the radiation of electromagnetic waves because of heat.

\subsubsection{Establishment of Heat Transfer Model in Three-Layer Fabrics}

Thermal protective clothing is made of three different layers of thermal protection materials, which is Layer I, Layer II and Layer III. In the process of heat transfer, when energy is transferred from the external environment to Layer I, it includes heat convection and heat radiation. Because Layer I can prevent most of the heat radiation, in the transmission of Layer II and Layer III, we neglect the heat radiation and only keep the heat conduction. Then, based on the Fourier's Law of Heat Conduction and previous studies (Torvi, 1997), the heat transfer model in three-layer fabrics is as follows :

$$
\left\{\begin{array}{l}
C_{I} \frac{\partial T}{\partial t}=\frac{\partial}{\partial x}\left(k_{I} \frac{\partial T}{\partial x}\right)+\gamma q_{\text {rad }} e^{-\gamma x}, x \in\left(0, L_{I}\right), t \in\left(0, t_{\text {end }}\right) \\
C_{I I} \frac{\partial T}{\partial t}=\frac{\partial}{\partial x}\left(k_{I I} \frac{\partial T}{\partial x}\right), x \in\left(L_{I}, L_{I}+L_{I I}\right), t \in\left(0, t_{\text {end }}\right) \\
C_{I I I} \frac{\partial T}{\partial t}=\frac{\partial}{\partial x}\left(k_{I I I} \frac{\partial T}{\partial x}\right), x \in\left(L_{I}+L_{I I}, L_{I}+L_{I I}+L_{I I I}\right), t \in\left(0, t_{\text {end }}\right)
\end{array}\right.
$$

Where $C_{I}, C_{I I}, C_{I I I}$ are the apparent heat capacity of Layer I, Layer II and Layer III respectively; $T$ is temperature; $t$ is time; $x$ is the distance from the left boundary of three-layer fabrics; $k_{I}, k_{I I}, k_{I I I}$ are the heat conductivity of Layer I, Layer II and Layer III respectively; $L_{I}, L_{I I}, L_{I I I}$ are the thickness of Layer I, Layer II and Layer III; $t_{\text {end }}$ is working time in high-temperature environment.

Based on the analysis of Torvi $(1997$; 1998), the apparent heat capacity is related with temperature. It is constant if the variation of ambient temperature is small (Chitrphiromsri \& Kuznetsov, 2005; Elgafy \& Mishra, 2014; Su, $\mathrm{He}, \& \mathrm{Li}, 2016)$, while in high-temperature environment, it can bring big errors if we still use constant value. In the heat transform model of three-layer fabrics, we introduce the apparent heat capacity method to show the variation of specific heat and reflect the energy variation of thermochemical reaction. The formula is as follows (Torvi, Eng., \& Threlfall, 2006):

$$
C=\rho c_{p}
$$

Where $\rho$ is the density of fabric; $c_{p}$ is the specific heat of fabric.

$q_{\text {rad }}$ is radiant heat from high-temperature environment to three-layer fabrics and it can be calculated by the following formula:

$$
q_{\text {rad }}=\sigma \varepsilon_{e} T_{e}^{4}-\sigma \varepsilon_{f a b} F_{f a b-s k i n}\left(1-\varepsilon_{e}\right)\left(T_{x=0}^{4}-T_{s k i n}^{4}\right)
$$

Where $\sigma$ is the Stefan-Boltzmann constant, equal to $5.67 \times 10^{-8} \mathrm{~W} / \mathrm{m}^{2} \cdot \mathrm{K}^{4} ; \varepsilon_{e}$ and $\varepsilon_{f a b}$ are the radiant emissivity of 
high-temperature environment and three-layer fabrics respectively; $F_{\text {fab-skin }}$ is the radiation shape factor of fabric radiation outward, equal to 1 (Kothandaraman, 2006); $T_{e}, T_{\text {fab }}, T_{\text {skin }}$ are the temperatures of high-temperature environment, three-layer fabrics and thermal manikin respectively.

$\gamma$ is the extinction coefficient of fabric and it can be calculated by the following formula:

$$
\gamma=\frac{\ln (\tau)}{L_{f a b}}
$$

Where $\tau$ is fabric projection rate.

\subsubsection{Establishment of Heat Transfer Model in Air Layer}

According to previous study (Ghazy \& Bergstrom, 2012), we can get the heat transfer model of the air layer:

$$
C_{I V} \frac{\partial T}{\partial t}=\frac{\partial}{\partial x}\left(k_{I V} \frac{\partial T}{\partial x}\right)-\frac{\partial q_{I V, r a d}}{\partial x}, x \in\left(L_{f a b}, L_{f a b}+L_{I V}\right), t \in\left(0, t_{\text {end }}\right)
$$

Some studies (Torvi, Dale, \& Faulkner, 1999) have shown that the heat transfer in the air layer is mainly heat radiation. Besides, when the thickness of air layer is less than $8 \mathrm{~mm}$, which is too small to form the heat convection, there is mainly heat conduction. Therefore, according to the principle of conductive and convective heat transfer in a limited space, we assume that there are only heat conduction in Layer IV and heat radiation on the surface of Layer IV. Then, the model of the air layer can be decoupled into the following formulas (Chitrphiromsri \& Kuznetsov, 2005; Lu, 2017):

$$
\begin{gathered}
-\left.k_{I V} \frac{\partial T_{I V}}{\partial x}\right|_{x=L_{f a b}}=-\left.k_{I V} \frac{\partial T_{I V}}{\partial x}\right|_{x=L_{f a b}+L_{I V}}=h_{c, a i r}\left(\left.T_{I I I}\right|_{x=L_{f a b}}-\left.T_{s k i n}\right|_{x=L_{f a b}+L_{I V}}\right) \\
\left.q_{I V, r a d}\right|_{x=L_{f a b}}=\left.q_{I V, r a d}\right|_{x=L_{f a b}+L_{I V}}=\frac{\sigma\left(\left.T_{I I I}^{4}\right|_{x=L_{f a b}}-\left.T_{s k i n}^{4}\right|_{x=L_{f a b}+L_{I V}}\right)}{\frac{1}{\varepsilon_{f a b}}+\frac{1}{\varepsilon_{s k i n}}-1}
\end{gathered}
$$

Where $q_{I V \text {,rad }}$ is the radiation flux of Layer IV; $\varepsilon_{f a b}, \varepsilon_{\text {skin }}$ are the radiant emissivity of Layer III and skin respectively.

$h_{c, a i r}$ is the heat transfer coefficient of air conduction and natural convection. The formula is:

$$
h_{c, a i r}=N u \frac{k_{\text {air }}(T)}{L_{\text {air }}}
$$

Where $N u$ is the Nusselt number, equal to 1 when natural convection is neglected.

The decoupled model in air layer simplifies the calculation and has good effect, so it has been used in many studies (Torvi, 1997; Song, 2003; Elgafy \& Mishra, 2014; Ghazy, 2014; Zhu, 2015).

\subsubsection{Determination of Initial Condition and Boundary Condition}

(1) Initial Condition

The initial condition is

$$
T(x, 0)=T_{I}(x)
$$

Where $T_{I}(x)$ is assumed to be $310.15 \mathrm{~K}$.

(2) Boundary Condition

The total heat flux to the surface of three-layer fabrics is the sum of radiant heat and convective heat, so the left boundary condition of the fabric layer is as follows (Torvi, 1997):

$$
-\left.k_{I} \frac{\partial T}{\partial x}\right|_{x=0}=\left.\left(q_{\text {conv }}+q_{\text {rad }}\right)\right|_{x=0}
$$

Where $q_{\text {conv }}$ is the convective heat from external environment to Layer I; $q_{\text {rad }}$ is the radiant heat from external environment to Layer I.

According to Chitrphiromsri and Kuznetsov (2005), we can convert the formula (10) into the following formula: 


$$
\left.\left(q_{c o n v}+q_{r a d}\right)\right|_{x=0}=h_{c, f a b}\left(T_{e}-\left.T_{I}\right|_{x=0}\right)
$$

Where $h_{c, f a b}$ is the heat transfer coefficient between external environment and Layer I; $T_{e}$ is the temperature of the external environment.

The boundary condition between Layer I and Layer II is:

$$
\left\{\begin{array}{l}
\left.T\right|_{x=L_{I}}=\left.T\right|_{x=L_{I}} \\
-\left.k_{I I} \frac{\partial T}{\partial x}\right|_{x=L_{I}}=-\left.k_{I} \frac{\partial T}{\partial x}\right|_{x=L_{I}}
\end{array}\right.
$$

The boundary condition between Layer II and Layer III is:

$$
\left\{\begin{array}{l}
\left.T\right|_{x=L_{I}+L_{2}}=\left.T\right|_{x=L_{I}+L_{2}} \\
-\left.k_{I I I} \frac{\partial T}{\partial x}\right|_{x=L_{I}+L_{I I}}=-\left.k_{I I} \frac{\partial T}{\partial x}\right|_{x=L_{I}+L_{I I}}
\end{array}\right.
$$

The right boundary condition of the fabric layer is as follows:

$$
-\left.k_{I I I} \frac{\partial T}{\partial x}\right|_{x=L_{f a b}}=\left.\left(q_{I V, r a d}-k_{I V} \frac{\partial T}{\partial x}\right)\right|_{x=L_{f a b}}
$$

Where $q_{I V, \text { rad }}$ is the radiant heat from the back of Layer III.

\subsection{Numerical Solution of Temperature Distribution for Heat Transfer Model}

The models we establish above is a whole, which involves four layers altogether from the left boundary of the thermal protective clothing to skin. According to this one-dimensional heat transfer model, there are all partial differential equations, which are difficult to solve. Therefore, we use the numerical method, the finite difference method, to discretize these partial differential equations and introduce the explicit difference schemes of them to simplify the calculation.

\subsubsection{Discretization of Partial Differential Equation}

(1) Explicit Difference Scheme for Three-Layer Fabrics

We discretize the continuous model by meshing the computational domain. Besides, we use forward difference for time and central difference for thickness. Then, we get the explicit difference scheme for three-layer fabrics as follows:

$$
\left\{\begin{array}{l}
C_{I} \frac{T_{j}^{i+1}-T_{j}^{i}}{\Delta t}=k_{I} \frac{T_{j+1}^{i}-2 T_{j}^{i}+T_{j-1}^{i}}{\Delta x^{2}}+\theta^{i}(x), x \in\left(0, L_{I}\right), t \in\left(0, t_{\text {end }}\right) \\
C_{I I} \frac{T_{j}^{i+1}-T_{j}^{i}}{\Delta t}=k_{I I} \frac{T_{j+1}^{i}-2 T_{j}^{i}+T_{j-1}^{i}}{\Delta x^{2}}, x \in\left(L_{I}, L_{I}+L_{I I}\right), t \in\left(0, t_{\text {end }}\right) \\
C_{I I I} \frac{T_{j}^{i+1}-T_{j}^{i}}{\Delta t}=k_{I I I} \frac{T_{j+1}^{i}-2 T_{j}^{i}+T_{j-1}^{i}}{\Delta x^{2}}, x \in\left(L_{I}+L_{I I}, L_{I}+L_{I I}+L_{I I I}\right), t \in\left(0, t_{\text {end }}\right)
\end{array}\right.
$$

Where $i, j$ is the step number of time and thickness respectively; $\Delta t, \Delta x$ is time step and thickness step respectively; $\theta^{i}(x)$ is the $i^{t h}$ step radiant heat.

Simplify these equations as follows:

$$
\left\{\begin{array}{l}
T_{j}^{i+1}=T_{j}^{i}+\frac{\mu k_{I}}{C_{I}}\left(T_{j+1}^{i}-2 T_{j}^{i}+T_{j-1}^{i}\right)+\frac{\theta^{i}(x) \Delta t}{C_{I}}, x \in\left(0, L_{I}\right), t \in\left(0, t_{\text {end }}\right) \\
T_{j}^{i+1}=T_{j}^{i}+\frac{\mu k_{I I}}{C_{I I}}\left(T_{j+1}^{i}-2 T_{j}^{i}+T_{j-1}^{i}\right), x \in\left(L_{I}, L_{I}+L_{I I}\right), t \in\left(0, t_{\text {end }}\right) \\
T_{j}^{i+1}=T_{j}^{i}+\frac{\mu k_{I I I}}{C_{I I I}}\left(T_{j+1}^{i}-2 T_{j}^{i}+T_{j-1}^{i}\right), x \in\left(L_{I}+L_{I I}, L_{I}+L_{I I}+L_{I I I}\right), t \in\left(0, t_{\text {end }}\right)
\end{array}\right.
$$

Where $\mu$ is as follows: 


$$
\mu=\frac{\Delta t}{\Delta x^{2}}
$$

(2) Explicit Difference Scheme for Air Layer

Because we assume that there are steady-state heat conduction in Layer IV and radiation heat transfer on the surface of Layer IV, the explicit difference scheme for air layer is:

$$
C_{I V} \frac{T_{j}^{i+1}-T_{j}^{i}}{\Delta t}=k_{I V} \frac{T_{j+1}^{i}-2 T_{j}^{i}+T_{j-1}^{i}}{\Delta x^{2}}, x \in\left(L_{f a b}, L_{f a b}+L_{I V}\right), t \in\left(0, t_{\text {end }}\right)
$$

\subsubsection{Discretization of Boundary Condition}

The following formulas in this section are explicit difference schemes for boundary conditions. The left boundary condition of the three-layer fabrics is:

$$
-k_{I} \frac{T_{1}^{i+1}-T_{0}^{i+1}}{d x}=h_{c, f a b}\left(T_{e}-T_{0}^{i+1}\right)
$$

The boundary conditions of the interface between three layers of fabric are as follows:

$$
\left\{\begin{array}{l}
-k_{I I} \frac{T_{L_{I}+1}^{i+1}-T_{L_{I}}^{i+1}}{d x}=-k_{I} \frac{T_{L_{I}}^{i+1}-T_{L_{I}-1}^{i+1}}{d x} \\
-k_{I I I} \frac{T_{L_{I}+L_{I I}+1}^{i+1}-T_{L_{I}+L_{I I}}^{i+1}}{d x}=-k_{I I} \frac{T_{L_{I}+L_{I I}}^{i+1}-T_{L_{I}+L_{I I}-1}^{i+1}}{d x}
\end{array}\right.
$$

The right boundary condition of the three-layer fabrics, which is also the left boundary condition of the air layer, is as follows:

$$
-k_{I I I} \frac{T_{L_{f a b}}^{i+1}-T_{L_{f a b}-1}^{i+1}}{d x}=h_{c, a i r}\left(T_{L_{f a b}}^{i+1}-T_{s k i n}\right)+\frac{\sigma\left(\left(T_{L_{f a b}}^{i+1}\right)^{4}-\left(T_{\text {skin }}\right)^{4}\right)}{\frac{1}{\varepsilon_{f a b}}+\frac{1}{\varepsilon_{\text {skin }}}-1}
$$

\subsubsection{Parameter Values of Experiment}

In order to test the performance of the heat transfer model, we apply it to analyzing the temperature distribution of the thermal protective clothing for high-temperature operation, which has been used for thermal manikin experiment. In the experiment, a thermal manikin, whose initial body temperature is $310.15 \mathrm{~K}$, wearing the thermal protective clothing, is placed in a laboratory with the ambient temperature at $348.15 \mathrm{~K}$ for 90 minutes, and the temperature of its lateral skin is taken at a regular time. The main parameter values of this experiment are shown in Table 2 and Table 3.

Table 2. Parameter Values of Thermal Protective Clothing Material

\begin{tabular}{ccccc}
\hline & $\rho\left(\mathrm{kg} / \mathrm{m}^{3}\right)$ & $c_{p}(\mathrm{~J} / \mathrm{kg} \cdot \mathrm{K})$ & $k(\mathrm{~W} / \mathrm{m} \cdot \mathrm{K})$ & $L(\mathrm{~mm})$ \\
\hline Layer I & 300 & 1377 & 0.082 & 0.6 \\
Layer II & 862 & 2100 & 0.37 & 6 \\
Layer III & 74.2 & 1726 & 0.045 & 3.6 \\
Layer IV & 1.18 & 1005 & 0.028 & 5 \\
\hline
\end{tabular}

Table 3. Other Parameter Values

\begin{tabular}{ccccc}
\hline$h_{c, f a b}\left(\mathrm{~W} / \mathrm{m}^{2} \cdot \mathrm{K}\right)$ & $\tau$ & $\varepsilon_{e}$ & $\varepsilon_{\text {fab }}$ & $\varepsilon_{\text {skin }}$ \\
\hline 120 & 0.01 & 0.02 & 0.9 & 0.95 \\
\hline
\end{tabular}

\subsubsection{Stability of Explicit Difference Equations}

The stability of explicit difference equations shows the ability of difference scheme to control error propagation in computation. The formula is as follows:

$$
0<\frac{k_{n} \Delta t}{C_{n} \Delta x^{2}}<\frac{1}{2}
$$


In order to satisfy the stability condition, ensure the convergence of the result and get the precise numerical solution, we determine $\Delta x=0.2$ and $\Delta t=0.0004$ according to the parameter values.

\section{Results and Discussions}

\subsection{Results of Temperature Distribution}

Based on the difference equations established above, we use $\Delta x=0.2$ and $\Delta t=0.0004$ to iterate. Then we can get the temperature of each fabric, that is, the temperature distribution of multilayer thermal protective clothing. According to the calculated data, the three dimensional temperature distribution can be plotted with MATLAB. The result is shown as Figure 2.
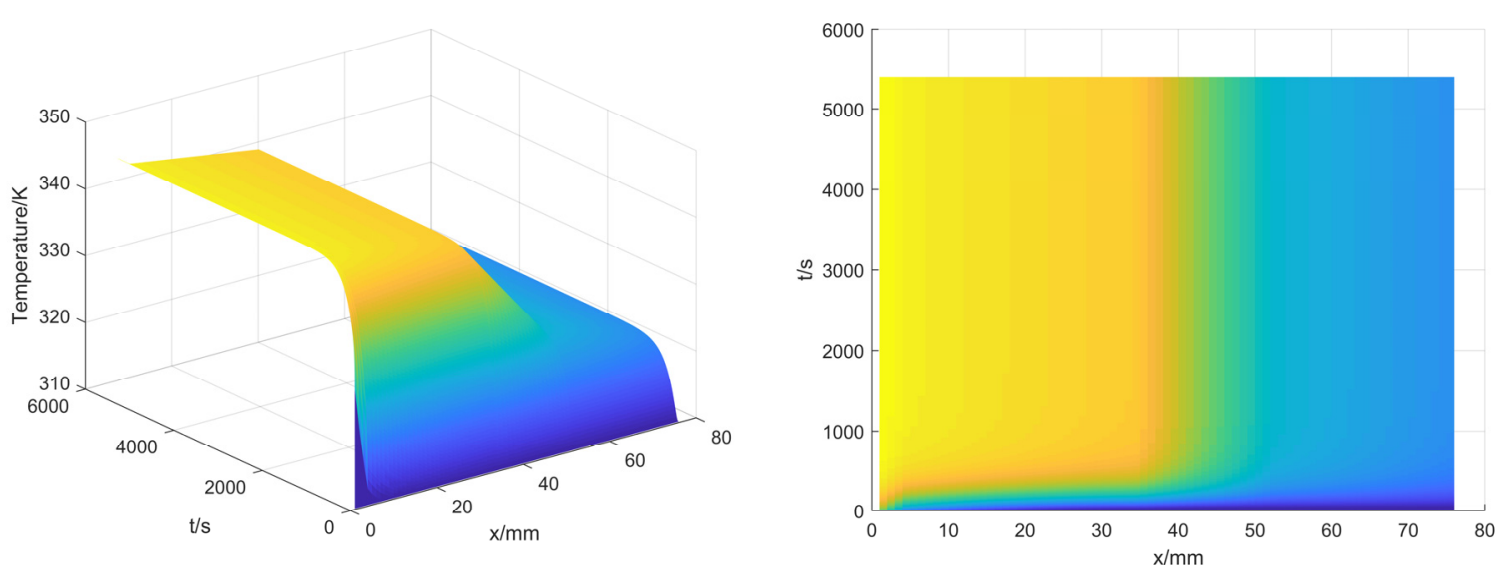

Figure 2. Three dimensional temperature distribution of thermal protective clothing

Using the control variable method, we control position or time invariant respectively to observe the variation of temperature with time at the same position or the variation of temperature with position at one moment. The results are as Figure 3 and Figure 4 and the steady-state temperature values are shown in Table 4.

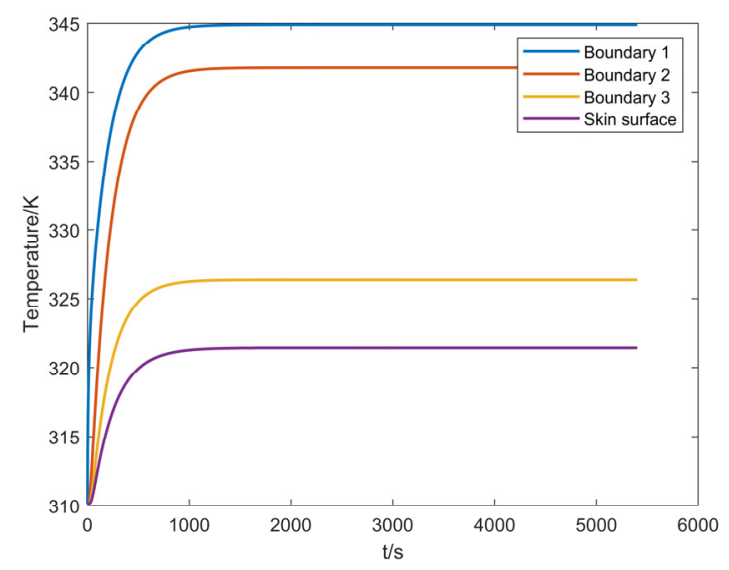

Figure 3. Temperature of boundary varies with time 


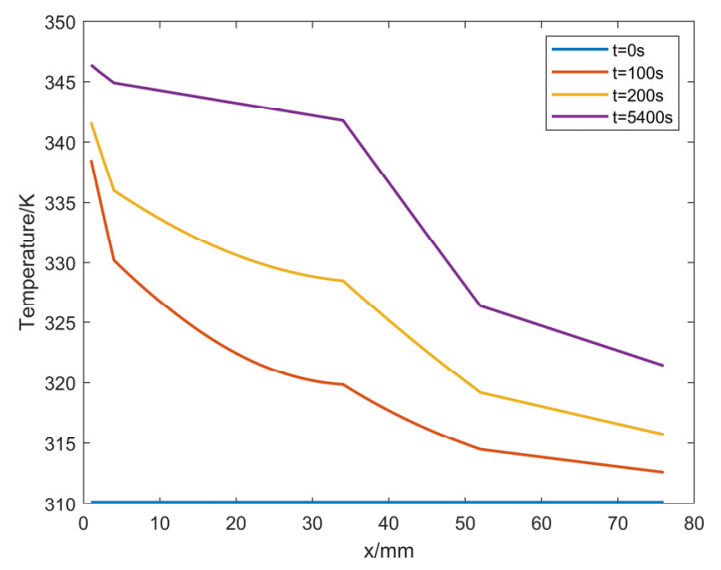

Figure 4. Temperature varies with thickness on different time

When it is steady-state, the temperatures of four layers are shown in Table 4.

Table 4. Convergence Values of Each Layer Temperature

\begin{tabular}{lllll}
\hline $\mathrm{t}(s)$ & $\mathrm{T}_{\mathrm{I}}(K)$ & $\mathrm{T}_{\text {II }}(K)$ & $\mathrm{T}_{\text {III }}(K)$ & $\mathrm{T}_{\text {IV }}(K)$ \\
\hline 5400 & 344.90 & 341.78 & 326.36 & 321.44 \\
\hline
\end{tabular}

According to Figure 3, when the thermal protective clothing is under the high-temperature environment, the temperature at the same position increases with time, and the temperature rising rate slows down gradually. Finally, it remains unchanged, which means that the heat transfer of each layer tends to be steady-state after a certain period of time. This is because the temperature difference between two points diminishes gradually, which results in the decrease of heat transfer rate. In addition, the trend of result data is the same as the temperature variation of the lateral skin measured in the experiment.

Then, Figure 4 shows that there are different temperature drop rates in different layers, which result in a discontinuity in term of gradient at boundaries. They are determined by the physical properties of the layers. The lower the thermal conductivity is, the better the heat insulation effect will be. It can be seen that the highest temperature drop rate appears between $34 \mathrm{~mm}$ and $52 \mathrm{~mm}$. It means that the fabric of best thermal protection performance is Layer III, which is known as the insulation layer.

During the experiment, the temperature of skin surface is measured per second. We compares the simulation result of skin surface with the experimental data, as shown in Figure 5.

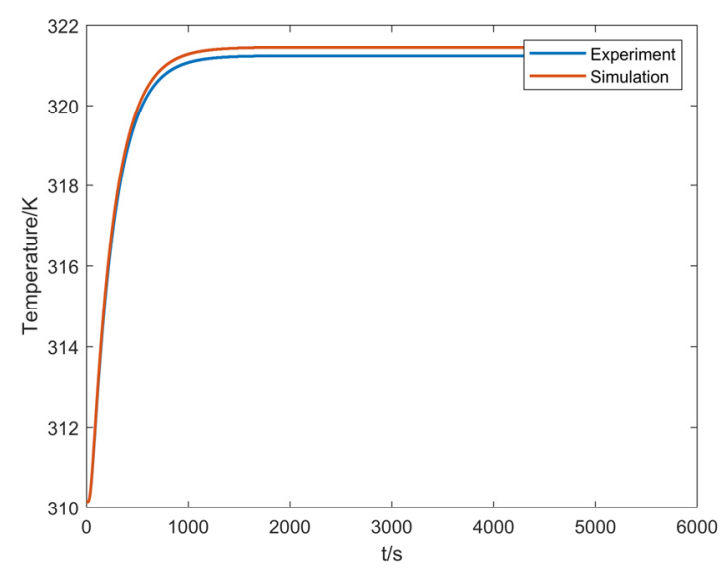

Figure 5. Comparison between simulation result and experimental data of skin surface

When heat transfer reaches steady state, the experimental data of skin surface is $321.23 \mathrm{~K}$ while the simulation result is about $321.435 \mathrm{~K}$. The error value is about $0.205 \mathrm{~K}$, which is acceptable. 


\subsection{Evaluation of Heat Transfer Model}

The heat transfer model constructed in this paper is a reference to the temperature distribution measurement of thermal protective clothing for high-temperature operation. It can be used to test the thermal protective performance of this clothing under specific environment and determine the critical value of applicable temperature for known material clothing, which provides guidance for the design of thermal protective clothing for high-temperature operation. In different working conditions, the fabric layer number of the model can be adjusted according to the actual situation, which makes the model more widely used.

However, this heat transfer model is an one-dimensional temperature conduction model, which only considers the temperature of the high-temperature environment, but neglects the impact of moisture transfer in different environmental humidity. In real application, according to the humidity characteristics of specific environment, this model can be adjusted and improved by introducing the moisture transfer, so that the model can be more practical and its role can be maximized.

\section{Conclusions}

In order to design more effective thermal protective clothing at less cost, based on the heat transfer theory and thermodynamics theory, we firstly establish heat transfer models of fabrics and air layer. In the three-layer fabrics, we consider the impact of heat conduction in three layers but only consider heat radiation in Layer I. Besides, we decouple and simplify the heat transfer model of Layer IV into a model with steady-state heat conduction in Layer IV and radiation heat transfer on surface of Layer IV. Secondly, using the finite difference method, we get the explicit difference schemes for the models, which can help to solve the partial differential equations. Then, we determine the time step and thickness step to ensure the stability of these explicit difference equations and get more precise results. According to the parameters and data collected in a thermal manikin experiment, we can solve the models and calculate the temperature of each layer. By simulation of MATLAB, the three-dimensional temperature distribution can be plotted. Finally, we compare the simulation result of skin surface with the experimental data. In conclusion, this study can provide reference for functional design of thermal protective clothing, and to some extent, it can reduce R\&D cost and shorten R\&D cycle.

\section{References}

Chitrphiromsri, P., \& Kuznetsov, A. V. (2005). Modeling heat and moisture transport in firefighter protective clothing during flash fire exposure. Heat and Mass Transfer, 41(3), 206-215. https://doi.org/10.1007/s00231-004-0504-x

Elgafy, A., \& Mishra, S. (2014). A heat transfer model for incorporating carbon foam fabrics in firefighter's garment. Heat \& Mass Transfer, 50(4), 545-557. https://doi.org/10.1007/s00231-013-1259-z

Ghazy, A. (2014). Numerical study of the air gap between fire-protective clothing and the skin. Journal of Industrial Textiles, 44(2), 257-274. https://doi.org/10.1177/1528083713483784

Ghazy, A., \& Bergstrom, D. J. (2010). Numerical simulation of transient heat transfer in a protective clothing system during a flash fire exposure. Numerical Heat Transfer, 58(9), 702-724. https://doi.org/10.1080/10407782.2010.516691

Ghazy, A., \& Bergstrom, D. J. (2012). Numerical simulation of heat transfer in firefighters $\mid$ " protective clothing with multiple air gaps during flash fire exposure. Numerical Heat Transfer, Part A: Applications, 61(8), 569-593. https://doi.org/10.1080/10407782.2012.666932

Ghazy, A., \& Bergstrom, D. J. (2013). Numerical simulation of the influence of fabric's motion on protective clothing performance during flash fire exposure. Heat and Mass Transfer, 49(6), 775-788. https://doi.org/10.1007/s00231-013-1123-1

Gibson, P. (1996). Multiphase heat and mass transfer through hygroscopic porous media with applications to clothing materials. Fiber, 53(5), 183-194. https://doi.org/10.2115/fiber.53.5_183

Kothandaraman, C. P. (2006). Fundamentals of Heat and Mass Transfer (3re ed.). New Delhi: New Age International Publishers.

Lawson, J. R., Mell, W. E., \& Prasad, K. (2010). A heat transfer model for firefighters' protective clothing, continued developments in protective clothing modeling. Fire Technology, 46(4), 833-841. https://doi.org/10.1007/s10694-010-0139-Z

Lu, L. Z. (2017). Mathematical model of heat transfer within multilayer thermal protective clothing and corresponding optional parameter determination (Master's thesis, Zhejiang Sci-Tech University, Zhejiang, 
China). Retrieved from https://www.cnki.net/

Mell, W. E. \& Lawson, J. R. (2000). A heat transfer model for firefighters' protective clothing. Fire Technology, 36(1), 39-68. https://doi.org/10.1023/A:1015429820426

Mercer, G. N., \& Sidhu, H. S. (2008). Mathematical modelling of the effect of fire exposure on a new type of protective clothing. Australian \& New Zealand Industrial \& Applied Mathematics Journal, 49, 289-305. https://doi.org/10.21914/anziamj.v49i0.346

Pan, B. (2016). Mathematical modeling of heat transfer within thermal protective clothing and inverse problems of parameters determination (Master's thesis, Zhejiang Sci-Tech University, Zhejiang, China). Retrieved from https://www.cnki.net/

Song, G. (2002). Modeling thermal protection outfits for fire exposures (Doctoral dissertation). Available from ProQuest Dissertation \& Theses: Full Text (3107792).

Song, G., Chitrphiromsri, P., \& Ding, D. (2008). Numerical simulations of heat and moisture transport in thermal protective clothing under flash fire conditions. International Journal of Occupational Safety and Ergonomics, 14(1), 89-106. https://doi.org/10.1080/10803548.2008.11076752

$\mathrm{Su}, \mathrm{Y} ., \mathrm{He}, \mathrm{J} .$, \& Li, J. (2016). Modeling the transmitted and stored energy in multilayer protective clothing under low-level radiant exposure. Applied Thermal Engineering, 93, 1295-1303. https://doi.org/10.1016/j.applthermaleng.2015.10.089

Torvi, D. A. (1997). Heat Transfer in Thin Fibrous Materials Under High Heat Flux Conditions (Doctoral dissertation, University of Alberta, Alberta, Canada). Retrieved from https://library.ualberta.ca/

Torvi, D. A., \& Dale, J. D. (1998). Effects of variations in thermal properties on the performance of flame resistant fabrics for flash fires. Textile Research Journal, 68(11), 787-796. https://doi.org/10.1177/004051759806801102

Torvi, D. A., Dale, J. D., \& Faulkner, B. (1999). Influence of Air Gaps On Bench-Top Test Results of Flame Resistant Fabrics. Journal of Fire Protection Engineering, 10(1), 1-12. https://doi.org/10.10.1177/104239159901000101

Torvi, D. A., Eng., P., \& Threlfall, T. G. (2006). Heat transfer model of flame resistant fabrics during cooling after exposure to fire. Fire Technology, 42(1), 27-48. https://doi.org/10.1007/s10694-005-3733-8

Zhu, F. L. (2015). Thermal Protection Function of Clothing. Beijing, BJ: China Textile \& Apparel Press.

\section{Copyrights}

Copyright for this article is retained by the author(s), with first publication rights granted to the journal.

This is an open-access article distributed under the terms and conditions of the Creative Commons Attribution license (http://creativecommons.org/licenses/by/4.0/). 